\title{
Patient reported outcome measures after incontinence and prolapse surgery: are the pictures painted by the ICIQ and PGI-I accurate?
}

\author{
Rufus Cartwright ${ }^{1} \cdot$ Heidi Brown ${ }^{2} \cdot$ Diaa Rizk $^{3}$
}

Received: 2 December 2015 / Accepted: 2 December 2015 /Published online: 11 January 2016

(C) The International Urogynecological Association 2015

Following surgery to treat stress urinary incontinence (SUI) or pelvic organ prolapse (POP), it is important to assess both objective and subjective outcomes. While not life-threatening, SUI and POP have potential for a significant adverse impact on quality of life, and thus the patient perspective has become increasingly important when evaluating treatment outcomes. Patient reported outcome measures (PROMs) are frequently used for subjective evaluation of the benefits of a treatment or intervention by gauging patients views on any change in their symptoms, function and health-related quality of life. In this issue, Larsen et al. report on the assessment of postoperative outcomes of SUI and POP surgery using PROMs [1].

It is commonly accepted in urogynaecological research and practice that different PROMs will measure different things. Although newly developed outcome measures are typically validated against a combination of existing objective and subjective measures, time and again, studies find limited correlations between different measures of disease severity or treatment improvement [2]. As a consequence, the available PROMs are often seen as complementary to one other. It is frequently recommended that clinicians should employ a range of PROMs, and it is usual for studies to employ a

Rufus Cartwright

r.cartwright@imperial.ac.uk

1 Department of Urogynaecology, Imperial College London, London, UK

2 Departments of Obstetrics \& Gynecology and Urology, Female Pelvic Medicine \& Reconstructive Surgery Section, Madison, WI, USA

3 Department of Obstetrics and Gynaecology, College of Medicine and Medical Sciences, Arabian Gulf University, Manama, Bahrain multitude of PROMs to fully capture different aspects of the patient experience of pelvic floor dysfunction [3, 4].

Larsen and colleagues compare the results of two different widely used PROMs. The International Consultation on Incontinence Questionnaire (ICIQ) system, developed from the earlier Bristol Female Lower Urinary Tract Symptoms (BFLUTS) questionnaire, measures the severity and bother of individual symptoms. In the format used in this study the ICIQ also provides separate global measures of interference in everyday life for urinary incontinence and POP. The Patient Global Impression of Improvement (PGI-I) is a seven-point scale that provides a single global measure of condition improvement. It was initially developed during the licensing trials of duloxetine for the treatment of SUI, but has subsequently been revalidated as a measure for improvement following treatment for POP and urgency incontinence.

Larsen et al.'s study has some remarkable features, most notably its sample size, with the underlying database including more than $90 \%$ of all surgeries performed for SUI and POP in Denmark during 2013, likely representing the largest ever reported surgical cohort study in urogynaecology. They find that overall results for both SUI and POP surgeries are excellent, with very substantial mean improvements on both the ICIQ and the PGI-I at the 3-month post-operative followup. The response rate of $60 \%$ also suggests some potential for bias, but regardless, these are largely reassuring data. Interestingly, the age trends in this study lead in the opposite directions for SUI and POP surgery, with younger women reporting better results for SUI surgery and worse results for POP surgery.

To make comparisons between the ICIQ and the PGI-I measures, individual scores for each were converted to the same scale. This procedure is likely fraught with difficulties, as the numerical values assigned to each category in the underlying items is somewhat arbitrary, and it is certainly 
questionable whether it makes sense to sum values obtained from the three ICIQ items. However, as in the original validation studies for the PGI-I, associations between measures (tested with weighted kappa) were fairly low, showing that they truly do test different aspects of treatment response. The authors found that the PGI-I tends to overstate the degree of improvement compared with the ICIQ, with a very large proportion of women selecting the maximum improvement category ("quite a lot better"). Worryingly, they find that the PGI-I correlates better with post-operative ICIQ scores than with change in ICIQ scores.

These findings may be simply due to recall bias, as the authors speculate, with women focused on their current levels of satisfaction, rather than the true degree of change due to surgery. We can also speculate, that the differences between the PGI-I and the ICIQ reflect true differences in what they measure. Many aspects of treatment response may not be reflected in the ICIQ items presented. Sexual function, bowel function, and other lower urinary tract symptoms may all be improved by POP surgery alone, but the questions asked on POP in the ICIQ reflect only the sensation of a lump in the vagina. Similarly, adverse effects of SUI surgery, such as de novo incontinence and persistent pain, are not tested by the ICIQ questions asked. Thus, it seems likely that the PGI-I might really provide a more global overview of treatment success, potentially more fully encompassing the range of benefits and harms of surgery.
Having raised a significant concern about an inherent bias in the PGI-I, the authors conclude that it should be used cautiously, and perhaps only in combination with other measures of treatment success. These findings should not detract from the importance of the PGI-I, or other global measures, as adjuncts to symptom-specific or disease-specific PROMs, which may miss important effects of complicated interventions, such as pelvic floor surgery.

\section{References}

1. Larsen MD, Lose G, Guldberg R, Gradel KO (2015) Discrepancies between patient reported outcome measures when assessing urinary incontinence or pelvis prolapse surgery. Int Urogynecol J DOI: 10. 1007/s00192-015-2840-4

2. Castillo PA, Espaillat-Rijo LM, Davila GW (2010) Outcome measures and definition of cure for female stress urinary incontinence surgery: a survey of recent publications. Int Urogynecol J 21:343348

3. Ghoniem G, Stanford E, Kenton K, Achtari C, Goldberg R, Mascarenhas $\mathrm{T}$ et al (2008) Evaluation and outcome measures in the treatment of female urinary stress incontinence: International Urogynecological Association (IUGA) guidelines for research and clinical practice. Int Urogynecol J Pelvic Floor Dysfunct 19:5-33

4. Tooz-Hobson P, Freeman R, Barber M, Maher C, Haylen B, Athanasiou $S$ et al (2012) An International Urogynecological Association (IUGA)/International Continence Society (ICS) joint report on the terminology for reporting outcomes of surgical procedures for pelvic organ prolapse. Int Urogynecol J 12:527-535 